\title{
Observation of the effect of physical rehabilitation therapy combined with the medication on pelvic floor dysfunction
}

\author{
WEI HAN ${ }^{1 *}$, YONGMEI WANG ${ }^{2 *}$, SHENGBO QI $^{3}$, TINGTING $\mathrm{LI}^{4}$, JIANG CAO $^{3}$, TINGHUA ZHENG ${ }^{5}$ and YAN $\mathrm{SU}^{6}$ \\ ${ }^{1}$ Maternity and Child Care Among Perinatal Care Clinic, Tangshan, Hebei 063000; ${ }^{2}$ Department of Gynecology \\ and Obstetrics, The Fourth Hospital of Hebei Medical University (Tumor Hospital of Hebei) Shijiazhuang, Hebei 050011; \\ ${ }^{3}$ Hospital of Gastrointestinal Surgery, Weifang, Shandong 261042; ${ }^{4}$ Department of Thoracic Surgery, Affiliated Hospital \\ of Hebei University, Baoding, Hebei 071000; ${ }^{5}$ Maternity and Child Care Department of Obstetrics and Gynecology; \\ ${ }^{6}$ Maternal and Child Health Care Medicine, Tangshan, Hebei 063000, P.R. China
}

Received February 22, 2017; Accepted May 18, 2017

DOI: $10.3892 /$ etm.2017.5583

\begin{abstract}
We observed the effects of physical rehabilitation therapy combined with medication on pelvic floor dysfunction (PFD). We collected the medical records of 84 maternal patients with PFD who received treatment at the Affiliated Hospital of Hebei University between May 2015 and October 2016. These patients were randomly divided into two groups, the control group $(n=42)$ and observation group $(n=42)$. In the control group, patients received conventional physical rehabilitation therapy while in observation group, patients received the shixiao powder combined with siwu decoction in addition to the physical rehabilitation. The therapeutic effects were compared between the two groups. The total effective rate of the observation group was $97.6 \%$, which was significantly higher than $78.6 \%$ in the control group $(\mathrm{P}<0.05)$. In observation group, the index scores of pelvic floor function under rest state, such as electromyo-graphy amplitude, contraction force, coordinate strength and urine flow rate, were significantly higher than those in control group $(\mathrm{P}<0.05)$. The rate of Grade II perineal muscle strength in the observation group was $7.32 \%$, which was significantly lower than $47.62 \%$ in control group $(\mathrm{P}<0.05)$. In observation group, the rates of Grade III and Grade IV muscle strength were respectively 43.91 and $29.26 \%$, which were significantly higher than those in control group $(\mathrm{P}<0.05)$. Before the administration of treatment, there were no significant differences in the comparison of the urinary incontinence and sexual life quality of patients between the two groups $(\mathrm{P}>0.05)$. In observation group, after
\end{abstract}

Correspondence to: Tingting Li, Department of Thoracic Surgery, Affiliated Hospital of Hebei University, 212 Yuhuadonglu, Baoding, Hebei 071000, P.R. China

E-mail: litingting201602@163.com

*Contributed equally

Key words: pelvic floor dysfunction, physical rehabilitation therapy, shixiao powder and siwu decoction, efficacy treatment, the rehabilitation effects of urinary incontinence and sexual life quality at 3 months post-delivery were significantly better than those in control group $(\mathrm{P}<0.05)$. Before treatment, there were no significant differences in the comparison of levels of C-reactive protein (CRP) and interleukin-10 (IL-10) between the two groups ( $\mathrm{P}>0.05)$, however, after treatment, the levels of CRP and IL-10 of patients in observation group were significantly lower than those in control group $(\mathrm{P}<0.05)$. In the observation group, the incidence rate of complications $(7.14 \%)$ were significantly lower than that in control group $(23.81 \%$; $\mathrm{P}<0.05)$. Therefore, physical rehabilitation therapy combined with the shixiao powder and siwu decoction exhibits significant efficacy in the treatment of PFD by effectively improving the pelvic floor functions, increasing perineal muscle strength, and decreasing expression levels of inflammatory factors, which can significantly ameliorate life quality and reduce the incidence rate of complications. Thus, this treatment method shows great application value in clinical practice.

\section{Introduction}

Pelvic floor dysfunction (PFD), a type of common gynecological disease in women, refers to the pelvic tissues that are affected by various pathogenesis and the external environment. This affects decreased immune ability and weakened structure, which leads to the susceptibility of pelvic organ displacement due to injured tissues, which leads to the abnormal location of organs and induces functional PFD (1-3). Clinical data (4) indicate that physical rehabilitation therapy, combined with herbal treatment, can effectively enhance pelvic functions and increase perineal muscle strength. Combination therapy, thus, represents a current trend in treatment of PFD. In this study, we mainly investigated the efficacy of physical rehabilitation therapy combined with medication on PFD.

\section{Patients and methods}

Sample selection. We collected the medical records of 84 maternal patients with PFD who received treatment at the Affiliated Hospital of Hebei University between May 2015 and October 2016. The inclusion criteria were as follows: 
i) patients were diagnosed with PFD in accordance to the relevant criteria (5); ii) patients with single pregnancy and without lochia on 42nd day after delivery; iii) patients with clear consciousness and good compliance; iv) patients without any diseases in major organs, such as the heart, liver and kidney and v) patients who signed the written informed consent. The exclusion criteria were as follows: i) patients who dropped out of this study, ii) patients with a history of pelvic operation and iii) patients with mental diseases.

A total of 84 patients were randomly divided into either the control group $(n=42)$ or the observation group $(n=42)$. In the control group, the ages of patients ranged from 22 to 35 years with an average of $28.62 \pm 3.17$ years. The disease course had lasted from 1-29 months with an average of $14.65 \pm 2.85$. In terms of the perineal muscle strength, there were 20 patients with Grade I, 17 with Grade II, 4 with Grade III and 1 with Grade IV. In the observation group, the ages of patients ranged from 23 to 36 years with an average of $29.43 \pm 3.29$ years. The disease course lasted from 2-31 months with an average of $15.89 \pm 2.96$. In terms of perineal muscle strength, there were 18 patients with Grade I, 20 with Grade II, 3 with Grade III, and 1 with Grade IV. The comparisons of the baseline material between the two groups showed no statistically significant differences $(\mathrm{P}>0.05)$. This study was approved by the Ethics Committee of the Affiliated Hospital of Hebei University. Signed written informed consents were obtained from all participants before the study.

Methods. In the control group, patients received conventional physical rehabilitation therapy. The physical rehabilitation therapy was conducted as follows: patients received a combination treatment of biological feedback and electrical stimulation via PHEMX. The load of treatment was adjusted according to the condition of patients. The treatment was conducted 2 times a week with 2-3 days in between, and lasted for a total of 2 courses ( 8 weeks/course). Next, training of pelvic floor was conducted as follows: after rehabilitation treatment, maternal patients were guided to perform an exercise of contraction of the pelvic muscle, i.e., contraction exercise of levator ani muscle, in which patients avoided the abdominal muscle being under stress. The exercise lasted for 10-15 min, and was carried out 2 times/day. Long-term training was considered.

In addition to the physical rehabilitation exercise that was the same as in the control group, patients in the observation group took shixiao powder and siwu decoction. The prescription included: $15 \mathrm{~g}$ of trogopterus dung; $12 \mathrm{~g}$ of rehmannia glutinosa; $12 \mathrm{~g}$ of angelica sinensis; $10 \mathrm{~g}$ carbo of typhae pollen, $9 \mathrm{~g}$ of ligusticum wallichii, $9 \mathrm{~g}$ of cyperus rotundus and $6 \mathrm{~g}$ of licorice. Drugs were soaked in water for $30 \mathrm{~min}$, which was followed by boiling on intense fire and decoction on slow fire for $15 \mathrm{~min}$. The methods of medication included 2 times/dose and 1 dose/day for a total of 3 weeks.

Observation and evaluation indexes. At the end of the third week in treatment, we observed the clinical efficacy of patients in both groups (6). Patients with Grade V muscle strength, 0 in fatigue, normal vaginal pressure and tension and elimination of symptoms was considered to be cured, while patients with an increase in muscle strength over 2 grades was deemed as effective (5).
At the end of third week in treatment, we also compared the pelvic floor function and grades of perineal muscle strength of patients between the two groups. The perineal muscle strength was graded in accordance with the following criteria (7). Grade I for perineal muscle that could not contract or could only contract for $1 \mathrm{sec}$. Grade II for perineal muscle that could perform incomplete contraction 2 times ( $2 \mathrm{sec} /$ time). Grade III for perineal muscle that could perform complete contraction 3 times ( $3 \mathrm{sec} /$ time). Grade IV for perineal muscle that could perform contraction 4 times (4 sec/time). Finally, Grade V for perineal muscle that could perform complete contraction 5 times or more (5 sec/time). Complications due to treatment included urinary incontinence, abscission of pelvic organs and anal dysfunction.

In addition, at the end of the third month after delivery, we observed the recovery of urinary incontinence and sexual life quality of patients of both groups (8).

Before treatment and at the end of the third week in the treatment, we collected $3 \mathrm{ml}$ of blood from each patient in order to assay for levels of inflammatory factors in serum via enzyme-linked immunosorbent assay (ELISA), which included C-reactive protein (CRP) and interleukin-10 (IL-10). Procedures were carried out in strict accordance to the instructions of the ELISA kits of CRP and IL-10 (Shanghai Lan School of Biological Science and Technology Co., Ltd., Shanghai, China). We observed the incidence of complications of patients in both groups during the treatment.

Statistical analysis. SPSS 20.0 software (IBM SPSS, Armonk, NY, USA) was adopted for statistical analysis. The measurement data are presented as mean \pm standard deviation. Chi-square test was adopted for comparison of count data, and paired-samples t-test for comparison of measurement data. $\mathrm{P}<0.05$ suggested that the difference was statistically significant.

\section{Results}

Comparison of clinical efficacy between the two groups. The total effective rate of observation group was $97.6 \%$, which was significantly higher than $78.6 \%$ in control group $(\mathrm{P}<0.05$; Table I).

Comparison of pelvic function of patients between the two groups. In the observation group, the index scores of pelvic floor function at rest state, such as the electromyography amplitude, contraction force, coordinate strength and urine flow rate, were significantly better than those in control group $(\mathrm{P}<0.05$; Table II).

Comparison of perineal muscle strength of patients between the two groups. The rate of Grade II perineal muscle strength in the observation group was $7.32 \%$, which was significantly lower than $47.62 \%$ in the control group $(\mathrm{P}<0.05)$. In observation group, the rates of Grade III and Grade IV muscle strength were, respectively, 43.91 and $29.26 \%$, which were significantly higher than those in the control group $(\mathrm{P}<0.05$; Table III).

Comparison of rehabilitation in urinary incontinence and sexual life quality of patients between the two groups. Before 
Table I. Comparison of clinical efficacy between the two groups (n).

\begin{tabular}{lccccc}
\hline Group & Case & Cure & Effective & Invalid & $\begin{array}{c}\text { Total effectiveness rate } \\
\text { of treatment }(\%)\end{array}$ \\
\hline Control group & 42 & 22 & 11 & 9 & 78.6 \\
Observation group & 42 & 34 & 7 & 1 & 97.6 \\
$\chi^{2}$ & & & & 9.016 \\
P-value & & & & $<0.05$ \\
\hline
\end{tabular}

Table II. Comparison of pelvic function of patients between the two groups (mean \pm standard deviation).

\begin{tabular}{lcccc}
\hline Group & $\begin{array}{c}\text { Electromyography } \\
\text { amplitude at rest }\end{array}$ & $\begin{array}{c}\text { Contraction } \\
\text { force }\end{array}$ & $\begin{array}{c}\text { Coordinate } \\
\text { strength }\end{array}$ & $\begin{array}{c}\text { Urine flow } \\
\text { rate }\end{array}$ \\
\hline Control group $(\mathrm{n}=42)$ & $17.59 \pm 6.83$ & $50.05 \pm 9.24$ & $3.27 \pm 1.36$ & $22.89 \pm 6.79$ \\
Observation group $(\mathrm{n}=42)$ & $7.52 \pm 4.23$ & $68.71 \pm 10.13$ & $6.27 \pm 1.75$ & $39.96 \pm 7.14$ \\
t-test & 8.445 & 9.165 & 10.032 & 11.728 \\
P-value & $<0.05$ & $<0.05$ & $<0.05$ & $<0.05$ \\
\hline
\end{tabular}

Table III. Comparison of perineal muscle strength of patients between the two groups [n (\%)].

\begin{tabular}{lccccc}
\hline Group & Grade I & Grade II & Grade III & Grade IV & Grade V \\
\hline Control group $(\mathrm{n}=42)$ & $8(19.05)$ & $20(47.62)$ & $12(28.57)$ & $2(4.76)$ & $1(2.38)$ \\
Observation group $(\mathrm{n}=42)$ & $2(4.88)$ & $3(7.32)^{\mathrm{a}}$ & $18(43.91)^{\mathrm{a}}$ & $12(29.26)^{\mathrm{a}}$ & $6(14.63)$ \\
$\chi^{2}$ & 0.165 & 8.326 & 7.094 & 8.815 & 0.094 \\
P-value & $>0.05$ & $<0.05$ & $<0.05$ & $<0.05$ & $>0.05$ \\
\hline
\end{tabular}

${ }^{\mathrm{a} C}$ Compared with control group, $\mathrm{P}<0.05$.

Table IV. Comparison of rehabilitation in urinary incontinence and sexual life quality of patients between the two groups (n).

\begin{tabular}{lccccc}
\hline & \multicolumn{2}{c}{ Urinary incontinence } & & \multicolumn{2}{c}{ Sexual life quality } \\
\cline { 2 - 3 } Group & Effective & Ineffective & & Effective & Ineffective \\
\hline Control group $(\mathrm{n}=42)$ & 34 & 8 & 33 & 9 \\
Observation group $(\mathrm{n}=42)$ & 41 & 1 & 39 & 3 \\
$\chi^{2}$ & 9.785 & 9.164 & & 10.907 & 7.821 \\
P-value & $<0.05$ & $<0.05$ & $<0.05$ & $<0.05$ \\
\hline
\end{tabular}

treatment, there were no significant differences in the comparison of urinary incontinence and sexual life quality of patients between the two groups $(\mathrm{P}>0.05)$. In the observation group, after treatment, the rehabilitation effects of urinary incontinence and sexual life quality in 3 months after delivery were significantly better than those in control group $(\mathrm{P}<0.05$; Table IV).

Comparisons of CRP and IL-10 levels before and after treatment of patients in the two groups. Before treatment, there was no significant difference in the comparison of the levels of CRP and IL-10 between the two groups $(\mathrm{P}>0.05)$.
After treatment, the levels of CRP and IL-10 of patients in the observation group were significantly lower than those in control group $(\mathrm{P}<0.05$; Table $\mathrm{V})$.

Comparison of incidence rates of complications between the two groups. In the observation group, the incidence rate of complications $(7.14 \%)$ were significantly lower than that in control group (23.81\%; $\mathrm{P}<0.05$; Table VI).

Analysis of a typical case. A female patient in the observation group aged 31 years, 12 months of disease course, and 
Table V. Comparisons of CRP and IL-10 levels before and after treatment of patients in two groups.

\begin{tabular}{lccrcc}
\hline & \multicolumn{2}{c}{ CRP $(\mathrm{mg} / \mathrm{l})$} & & \multicolumn{2}{c}{$\mathrm{IL}-10(\mu \mathrm{g} / \mathrm{l})$} \\
\cline { 2 - 3 } Group & Before treatment & After treatment & & Before treatment & After treatment \\
\hline Control group $(\mathrm{n}=42)$ & $81.54 \pm 8.93$ & $42.77 \pm 4.45$ & & $105.92 \pm 12.52$ & $68.32 \pm 5.66$ \\
Observation group $(\mathrm{n}=42)$ & $81.64 \pm 9.12$ & $36.05 \pm 4.03$ & & $106.73 \pm 11.93$ & $54.41 \pm 7.02$ \\
t-test & 0.172 & 9.116 & & 0.115 & 10.773 \\
P-value & $>0.05$ & $<0.05$ & & $>0.05$ & $<0.05$ \\
\hline
\end{tabular}

CRP, C-reactive protein; IL-10, interleukin-10.

Table VI. Comparison of incidence rates of complications between the two groups [n (\%)].

\begin{tabular}{lccc}
\hline Group & $\begin{array}{c}\text { Urinary } \\
\text { incontinence }\end{array}$ & $\begin{array}{c}\text { Abscission of } \\
\text { pelvic organs }\end{array}$ & $\begin{array}{c}\text { Anal } \\
\text { dysfunction }\end{array}$ \\
\hline Control group $(\mathrm{n}=42)$ & $4(9.52)$ & $3(7.14)$ & $3(7.14)$ \\
Observation group $(\mathrm{n}=42)$ & $2(4.76)$ & $1(2.38)$ & $0(0)$ \\
$\chi^{2}$ & & & $3(7.14)$ \\
P-value & & & 12.531 \\
\end{tabular}

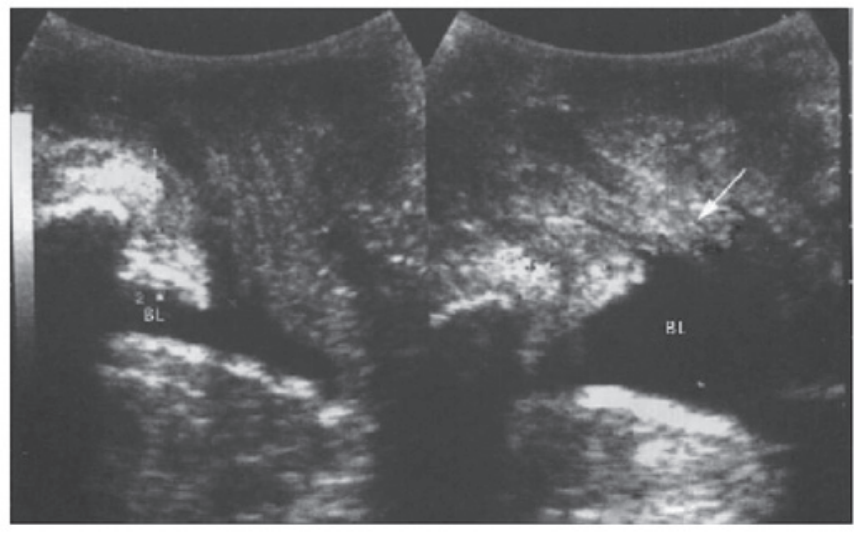

Figure 1. On the left, rest state; on the right, Valsalva action. Arrow indicates the choanoid urinary tract.

Grade III pelvic muscle strength, had the clinical manifestation of urinary incontinence. Before treatment, the ultrasonic examination showed the choanoid urinary tract (Fig. 1), Grade II cystocele and Grade II prolapse of uterus (Fig. 2). After the combination treatment of physical rehabilitation therapy and shixiao powder and siwu decoction, this patient was cured without any complications in treatment.

\section{Discussion}

Studies have shown that PFD is characterized by a high incidence rate $(9,10)$. At delivery, there is an excessive pull of pelvic muscle, ligament and fascia tissue, which can injure the perineal tendon at the attachment point of pelvic muscle, resulting in an alteration of attachment point, decrease in innervation ability and reduction in pelvic muscle strength,

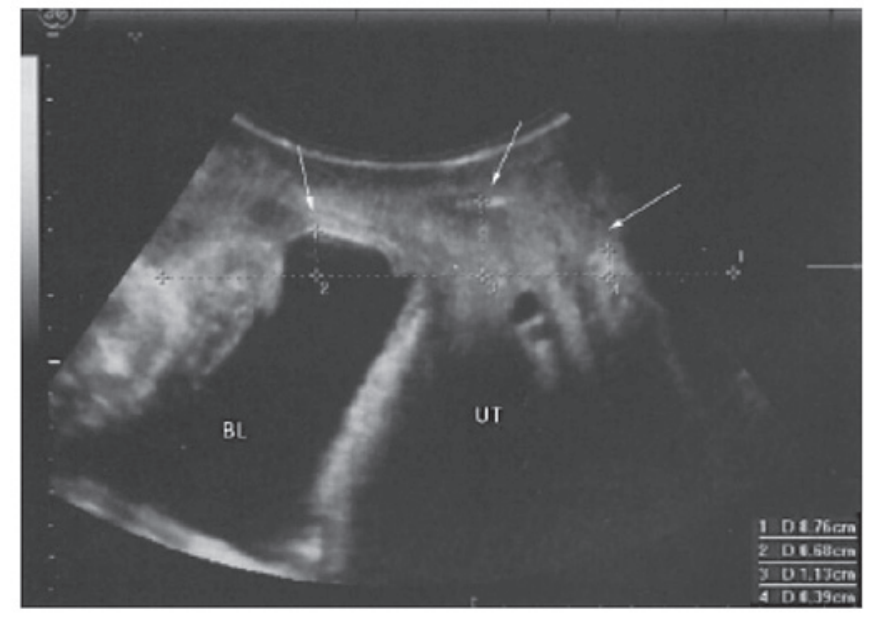

Figure 2. Grade II, cystocele and Grade II, prolapse of uterus. Arrows indicate cystocele and prolapse.

causing further dysfunction. PFD can induce urinary dysfunction of patients, giving rise to the abscission of pelvic organs, stress urinary incontinence and anal dysfunction and thus, the patients' regular life can be severely affected (11-13).

Physical rehabilitation therapy refers to a treatment pattern in which the PHEMX machine is applied in order to perform electrical stimulation according to the biological response of the body and physical rehabilitation therapy, with advantages that are non-invasive, painlessness, with fewer adverse reactions and significant efficacy, effectively ameliorating the pelvic functions and the clinical symptoms of maternal women (14-17). However, since physical rehabilitation therapy only relies on current feedback and exercise of pelvic muscle, it is susceptible to therapeutic errors which can decrease the 
medical consultation rate and affect the continuity of treatment. Therefore, for patients in the observation group, we applied shixiao powder and siwu decoction in addition to the physical rehabilitation therapy, which aims, based on Chinese Medicine, to increase the therapeutic efficacy and promote the improvement in conditions of patients. In this prescription, compatible application of rehmannia glutinosa (for nourishing yin, tonifying blood, enriching essence and marrow), ligusticum wallichii (promoting the circulation of $q i$ and blood), cyperus rotundus (for soothing the liver and regulating vital energy), licorice (clearing heat and tonifying qi, and regulating herbal property), and other drugs can effectively ameliorate the symptoms of patients after delivery, such as qi stagnation and blood stasis and blood loss, which reaches the goal of invigorating spleen-stomach and replenishing qi, enriching blood, promoting blood flow, strengthening body resistance and eliminating evil, thus accelerating the treatment and promoting the rehabilitation of patients $(10,18,19)$. According to modern pharmacology $(20,21)$, herbal treatment can promote the blood circulation of perineal tissues, eliminate symptoms of qi stagnation and blood stasis and enhance pelvic muscle strength to prompt the self-recovery of connective tissue, fascia tissue and ligament tissue and increase muscle tone. Thus, herbal treatment, plus physical stimulation and exercise of pelvic functions, can rapidly increase the rehabilitation efficiency of pelvic function, and decrease the incidence rate of urinary incontinence, anal dysfunction and abscission of pelvic tissues.

The results of this study revealed that the total effective rate of observation group was $97.6 \%$, which was significantly higher than the $78.6 \%$ in control group $(\mathrm{P}<0.05)$. In the observation group, the index scores of pelvic floor function under a rest state, such as electromyography amplitude, contraction force, coordinate strength and urine flow rate, were significantly improved when compared to the control group $(\mathrm{P}<0.05)$. The rate of Grade II perineal muscle strength in the observation group was $7.32 \%$, which was significantly lower than $47.62 \%$ in the control group $(\mathrm{P}<0.05)$. In the observation group, the rates of Grades III and IV muscle strength were, respectively, 43.91 and $29.26 \%$, which were significantly higher than those in control group $(\mathrm{P}<0.05)$. In the observation group, after treatment, the rehabilitation effects of urinary incontinence and sexual life quality in 3 months post-delivery were significantly better than those in control group $(\mathrm{P}<0.05)$. In the observation group, the incidence rate of complications $(7.14 \%)$ was significantly lower than those in the control group $(23.81 \%$; $\mathrm{P}<0.05)$. These results confirm that physical rehabilitation therapy combined with shixiao powder and siwu decoction can significantly ameliorate pelvic function, increase perineal muscle strength and decrease the incidence rate of complication in patients.

Inflammatory factors, such as CRP and IL-10, were positively involved in the occurrence and development of PFD (22). In patients with PFD, the levels of inflammatory factors in the body, such as CRP and IL-10, were significantly higher than those in healthy women (15). In this study, we found that before treatment, there were no significant differences in comparisons of levels of CRP and IL-10 between the groups $(\mathrm{P}>0.05)$. However, after treatment, the levels of CRP and IL-10 of patients in observation group were significantly lower than those in control group $(\mathrm{P}<0.05)$. These results suggest that the physical rehabilitation therapy combined with shixiao powder and siwu decoction can significantly decrease the levels of inflammatory factors, and the potential mechanism may be that physical rehabilitation therapy combined with shixiao powder and siwu decoction can continuously activate the bioactive molecules to increase the quantity of myocytes, promote blood circulation, effectively regulating venous return and reduces the accumulation of various harmful metabolites and inflammatory factors, such as CRP and IL-10.

In conclusion, physical rehabilitation therapy combined with the shixiao powder and siwu decoction exhibits significant efficacy in the treatment of PFD by effectively improving pelvic floor functions, increasing perineal muscle strength, and decreasing expression levels of inflammatory factors, which can significantly ameliorate quality of life and reduce the incidence rate of complications. Thus, this treatment method shows great application value in clinical practice.

\section{References}

1. Bedere N, Delaby L, Ducrocq V, Leurent-Colette S and Disenhaus C: Toward improved postpartum cyclicity of primiparous dairy cows: effects of genetic merit for production traits under contrasting feeding systems. J Dairy Sci 99: 1266-1276, 2016.

2. Woolhouse H, James J, Gartland D, McDonald E and Brown SJ: Maternal depressive symptoms at three months postpartum and breastfeeding rates at six months postpartum: implications for primary care in a prospective cohort study of primiparous women in Australia. Women Birth 29: 381-387, 2016.

3. Tsuchiya M, Mori E, Sakajo A, Maekawa T, Iwata H, Maehara K, Morita A, Ozawa H, Mochizuki Y, Aoki K, et al: Age-specific determinants of post-partum fatigue in primiparous women. Jpn J Nurs Sci 13: 83-94, 2016.

4. Durnea CM, Khashan AS, Kenny LC, Tabirca SS and O'Reilly BA: The role of prepregnancy pelvic floor dysfunction in postnatal pelvic morbidity in primiparous women. Int Urogynecol J Pelvic Floor Dysfunct 25: 1363-1374, 2014.

5. Gaspard L, Tombal B, Castille Y, Opsomer RJ and Detrembleur C: Pelvic floor muscles training, electrical stimulation, bladder training and lifestyle interventions to manage lower urinary tract dysfunction in multiple sclerosis: a systematic review. Prog Urol 24: 222-228, 2014 (In French).

6. Murad-Regadas SM, Regadas FilhoFS, Regadas FS, Rodrigues LV, de J R Pereira J, da S Fernandes GO, Dealcanfreitas ID and Mendonca Filho JJ: Use of dynamic 3-dimensional transvaginal and transrectal ultrasonography to assess posterior pelvic floor dysfunction related to obstructed defecation. Dis Colon Rectum 57: 228-236, 2014.

7. Luthander C, Emilsson T, Ljunggren G and Hammarström M: A questionnaire on pelvic floor dysfunction postpartum. Int Urogynecol J Pelvic Floor Dysfunct 22: 105-113, 2011.

8. Pannu HK, Javitt MC, Glanc P, Bhosale PR, Harisinghani MG, Khati NJ, Mitchell DG, Nyberg DA, Pandharipande PV, Shipp TD, et al: ACR appropriateness criteria pelvic floor dysfunction. J Am Coll Radiol 12: 134-142, 2015.

9. Bondurri A, Maffioli A and Danelli P: Pelvic floor dysfunction in inflammatory bowel disease. Minerva Gastroenterol Dietol 61: 249-259, 2015.

10. Erekson EA, Fried TR, Martin DK, Rutherford TJ, Strohbehn K and Bynum JPW: Frailty, cognitive impairment, and functional disability in older women with female pelvic floor dysfunction. Int Urogynecol J Pelvic Floor Dysfunct 26: 823-830, 2015.

11. Sighinolfi MC, Rivalta M, Mofferdin A, Micali S, De Stefani S and Bianchi G: Potential effectiveness of pelvic floor rehabilitation treatment for postradical prostatectomy incontinence, climacturia, and erectile dysfunction: a case series. J Sex Med 6: 3496-3499, 2009.

12. Ramalingam $\mathrm{K}$ and Monga A: Obesity and pelvic floor dysfunction. Best Pract Res Clin Obstet Gynaecol 29: 541-547, 2015.

13. Jundt K, Peschers U and Kentenich $H$ : The investigation and treatment of female pelvic floor dysfunction. Dtsch Arztebl Int 112: 564-574, 2015. 
14. Tibaek S and Dehlendorff C: Pelvic floor muscle function in women with pelvic floor dysfunction: a retrospective chart review, 1992-2008. Int Urogynecol J Pelvic Floor Dysfunct 25: 663-669, 2014.

15. Bezerra LR, Vasconcelos Neto JA, Vasconcelos CT, Karbage SA, Lima AC, Frota IP, Rocha AB, Macedo SR, Coelho CF, Costa MK, et al: Prevalence of unreported bowel symptoms in women with pelvic floor dysfunction and the impact on their quality of life. Int Urogynecol J 25: 927-933, 2014.

16. Wen Y, Polan ML and Chen B: Do extracellular matrix protein expressions change with cyclic reproductive hormones in pelvic connective tissue from women with stress urinary incontinence? Hum Reprod 21: 1266-1273, 2006.

17. Bortolami A, Vanti C, Banchelli F, Guccione AA and Pillastrini P Relationship between female pelvic floor dysfunction and sexual dysfunction: an observational study. J Sex Med 12: 1233-1241, 2015.

18. Sangsawang B and Sangsawang N: Stress urinary incontinence in pregnant women: a review of prevalence, pathophysiology, and treatment. Int Urogynecol J 24: 901-912, 2013.

19. Bozkurt M, Yumru AE and Şahin L: Pelvic floor dysfunction, and effects of pregnancy and mode of delivery on pelvic floor. Taiwan J Obstet Gynecol 53: 452-458, 2014.
20. Capobianco G, Wenger JM, Meloni GB, Dessole M, Cherchi PL and Dessole S: Triple therapy with Lactobacilli acidophili, estriol plus pelvic floor rehabilitation for symptoms of urogenital aging in postmenopausal women. Arch Gynecol Obstet 289: 601-608, 2014.

21. van Delft K, Sultan AH, Thakar R, Schwertner-Tiepelmann N and Kluivers K: The relationship between postpartum levator ani muscle avulsion and signs and symptoms of pelvic floor dysfunction. BJOG 121: 1164-1171, discussion 1172, 2014.

22. Rosenkrantz AB, Lewis MT, Yalamanchili S, Lim RP, Wong S and Bennett GL: Prevalence of pelvic organ prolapse detected at dynamic MRI in women without history of pelvic floor dysfunction: comparison of two reference lines. Clin Radiol 69: e71-e77, 2014. International (CC BY-NC-ND 4.0) License. 